\title{
Luminescence from quantum wells arranged within a microcavity
}

\author{
A. FREY, G. JUNGK and R. HEY \\ Paul-Drude-Institut für Festkörperelektronik, Hausvogteiplatz 5-7, 10117 Berlin, Germany
}

\begin{abstract}
Detailed investigations are presented of photoluminescence spectra from two slightly different and wedge-shaped InGaAs quantum wells (QWs) positioned at the antinode and near the node of a GaAs $\lambda / 2$-cavity, where $n \lambda=\lambda_{0}$ is the emission wavelength in vacuum and $n$ the refractive index. The measurements are performed at $5 \mathrm{~K}$. Position dependences show energy shifts of the luminescence lines due to the intended shape of the QWs and a variation of the intensities. Angle dependences show distinct deviations from the $\cos ^{2}$ - law of free dipol radiation as well as from the modified radiation pattern in a resonator. For an emission wavelength below the resonator mode the intensity peaks at a $30^{\circ}$ inclination from the surface normal. Investigations of the resonator's reflectivity reveal its sensitivity against absorption. Ellipsometric determination of the effective dielectric function results in the absolute reflectivity of the constituting Bragg reflectors up to $99.8 \%$ at the stop-band. This proves the enhanced sensitivity of the resonator's optical response on tiny variations of the optical and geometrical parameters. The exact coincidence of the emitted wavelength and the resonator mode was not achieved for emission parallel to the surface normal, the emission is not phase correlated.
\end{abstract}

\section{Background}

The elementary interaction between an atom and the photon field is strongly modified, when the atom is confined to a resonant cavity. Enhancement as well as inhibition of the spontaneous emission is observed, Rabi-splitting and line-width variation are predicted from QED calculations and measured in careful experiments. However, the observations can also be interpreted classically, taking into account the Kramers-Kronig related refraction and absorption of photons through the atoms within the cavity [1 - 4]. Similar experiments on the luminescence of QWs placed within a cavity or from microcrystals reveal an analogous ensemble of effects, where the energy shift of optical transitions is often relatedto quantummechanical confinement effects. It is interesting to note, that "classical" interpretations are also here successful, where interference, the photonic density of states and local field effects due to different [5 - 
8]. Here we search for cavity effects on the spontaneous emission of QWs, i. e. the influence of the resonator mode on intensity, energy, line-width and coherence of the luminescence radiation.

\section{Experimental}

The MBE-grown sample configuration is shown in Fig. 1. Two Bragg-reflectors made from 18 pairs $\mathrm{Al}_{0.3} \mathrm{Ga}_{0.7} \mathrm{As} / \mathrm{GaAs}$ and 15 pairs $\mathrm{AlAs} / \mathrm{GaAs}$, resp. limit the $\mathrm{GaAs}$-resonator. The films are deposited with rotating sample holder on a semiinsulating (100) oriented GaAs-substrate. Only for the deposition of the $\mathrm{In}_{0.1} \mathrm{Ga}_{0.9}$ As-QWs the rotation was stopped. Therefore we got highly uniform film-thicknesses and slightly wedge-shaped wells.

Due to the material combination the emission energy is below the absorption edge of the embedding materials. The QWs are placed at the antinode and near the node of the resonator, their thicknesses are 7 $\mathrm{nm}$ and $5 \mathrm{~nm}$, resp., therefore they can simply be distinguished by the emission wavelength. The photoluminescence was excited with the $1.96 \mathrm{eV}$ line of a He-Ne laser. The measurements have been performed at $5 \mathrm{~K}$ with the propagation vector of the exciting and luminescence radiation nearly antiparallel. Position- and angle-dependences could be recorded through variation of the excitation spot position and sample rotation around an axis perpendicular to the plane of incidence and the surface normal.

The reflectivity (in arbitrary units) was measured in the same apparatus also at $5 \mathrm{~K}$. The absolute value of the reflectivity was determined from ellipsometric investigations with a variable angle of incidence ellipsometer at room-temperature. For the reflector's stop-band the mirror behaves effectively as a Drude metal, whose ellipsometric parameters can be determined precisely enough, when the interaction is used to produce circularly polarized reflected radiation.

\section{Results and discussion}

Fig. 2 presents the lateral distribution of the photoluminescence from a rough line-scan along the resonator structure. Shifts of the luminescence peaks' energies are due to the intended wedge shape of the QWs and to unavoidable variations of the chemical composition of the well material. The strong intensity modulation is related to varying excitation- and/or emission conditions. They give relative advantage to the luminescence from the QW nearer to the node.

In Fig. 3 the reflectivity (normalized to one) and a luminescence spectrum are compared. The ellipsometric investigations result in absolute reflectivities up to $99.8 \%$ i. e. $F=4 R(1-R)^{-2} \approx 10^{6}$. Therefore, small fractions of absorbed light $\mathrm{A}$ are detected in the reflectivity experiments, due to [9]

$$
\mathrm{I}_{\text {refl. }}=\mathrm{I}_{\text {inc }} \frac{F \sin ^{2} \frac{\delta}{2}(1-A)+A^{2}}{1+F \sin ^{2} \frac{\delta}{2}}
$$

with $\delta=4 \pi \mathrm{h} \lambda_{\mathrm{o}}-1 \sqrt{n^{2}-\sin ^{2} \gamma}$, where $\mathrm{h}$ denotes the geometrical cavity length. 
Consequently in addition to the resonator mode near $1.505 \mathrm{eV}$ we observe minima at $1.460,1.474$ and $1.515 \mathrm{eV}$, which are connected with the HH1E1 transition in the QWs and the excitonic transition in GaAs, resp.

With regard to the angle-dependences we found deviations from the resonator influenced radiation pattern calculated from [10]

$$
I_{e m} \sim(1-R) \frac{1+R+\sqrt{R} \cos z}{(1-R)^{2}+4 R \sin ^{2} z} \begin{cases}\sin ^{2} \psi & s-\text { wave } \\ \cos ^{2} \psi \cos ^{2} \psi & p \text {-wave }\end{cases}
$$

with $z=(\cos \gamma+1) \pi$ and $\psi$ denotes the analyser azimuth relative to the plane of incidence. Fig. 4 gives experimental data for both QWs and a normalized spectrum calculated from Eq. (2) assuming $R=0.90$. The experimentally determined increased emission from the $5 \mathrm{~nm}$ QW at $\gamma=30^{\circ}$ could be due to interaction between both wells. Because of $\lambda_{0}>\mathrm{nh}$ no angle-fitting to the resonator mode is possible.

1 S. Haroche, Physics World 4, 33 (March 1991)..

2 M. G. Raizen, R. J. Thompson, R. J. Brecha, H. J. Kimble, and H. J. Carmichael, Phys. Rev. Letters 63,240 (1989).

3 Yifu Zhu, Daniel J. Gauthier, S. E. Morin, Quilin Wu, H. J. Carmichael, and T. W. Mossberg, Phys. Rev. Letters 64, 2499 (1990).

4 H. J. Carmichael, Phys. Rev. A 44, 4751 (1991).

5 G. Jungk and U. Woggon, Superlattices and Microstructures 9,401 (1991).

6 R. J. Horowicz, H. Heitmann, Y. Kadota, and Y. Yamamoto, Appl. Phys. Letters 61, 393 (1992).

7 C. Lei, Z. Huang, D. G. Deppe, C. J. Pinzone, and R. D. Dupuis, J. Appl. Phys. 73 , 2700 (1993).

8 S. Juen, K. Überbacher, J. Baldauf, K. F. Lamprecht, and R. A. Höpfel, Appl. Phys. Letters 62, 1958 (1993)

9 M. Born, E. Wolf, Principles of Optics, Pergamon Press (6. Ed. 1986) chapter 7.6

10 Y. Yamamoto, S. Machida, K. Igeta, and G. Björk in: Coherence, Amplification, and Quantum Effects in Semiconductor Lasers (ed. by Y. Yamamoto), J. Wiley \& Sons, Inc. New York, 1991 


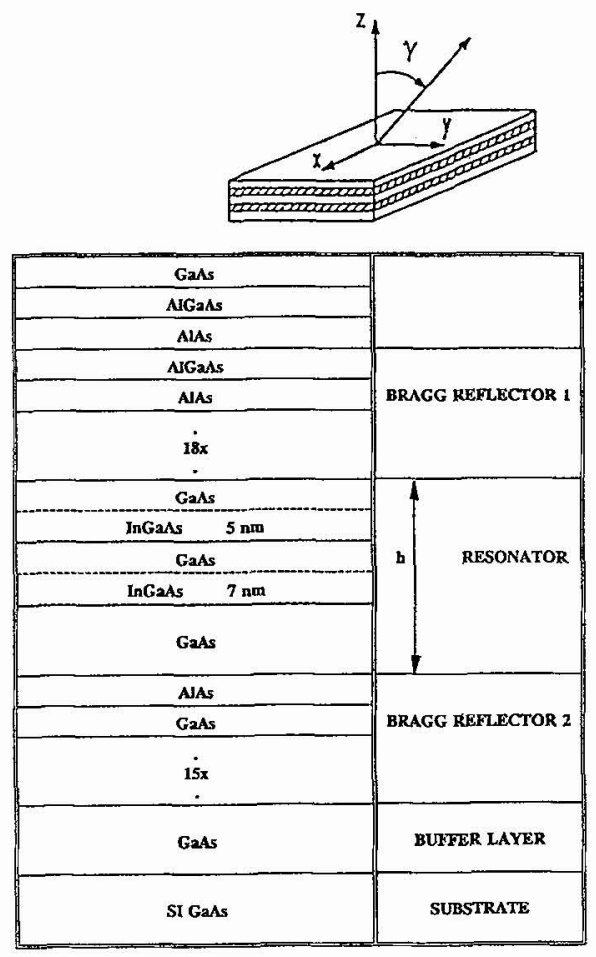

Fig.1 Sample design and its orientation in angle dependent measurements.

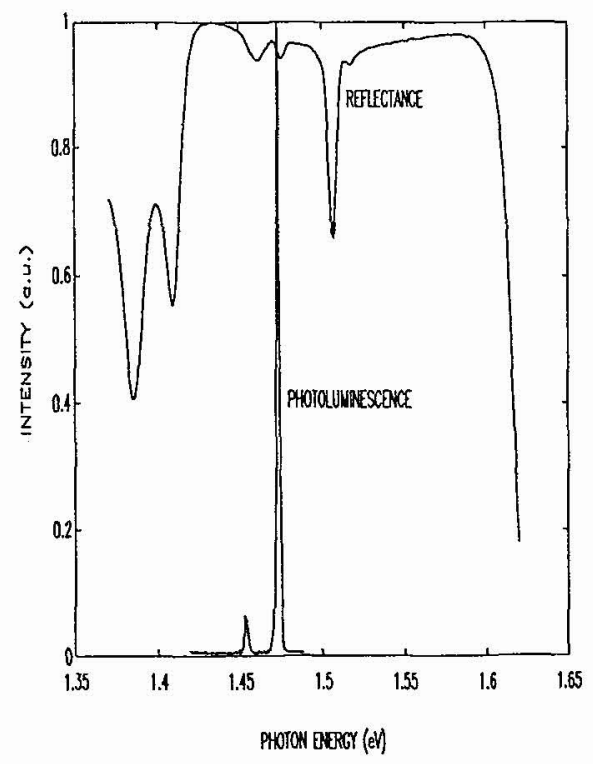

Fig.3 Reflectance and photoluminescence at $5 \mathrm{~K}$.

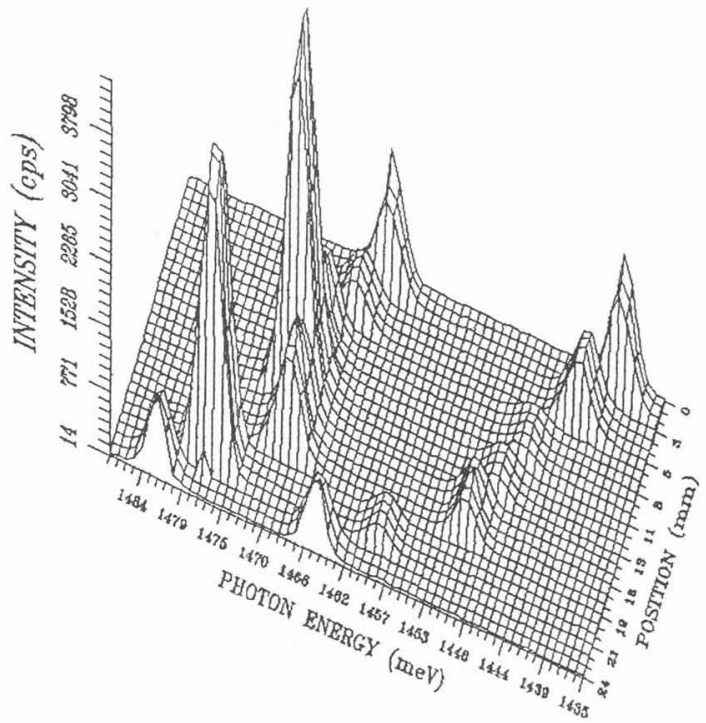

Fig.2 Lateral distribution of the photo-luminescence normal to the sample's surface.

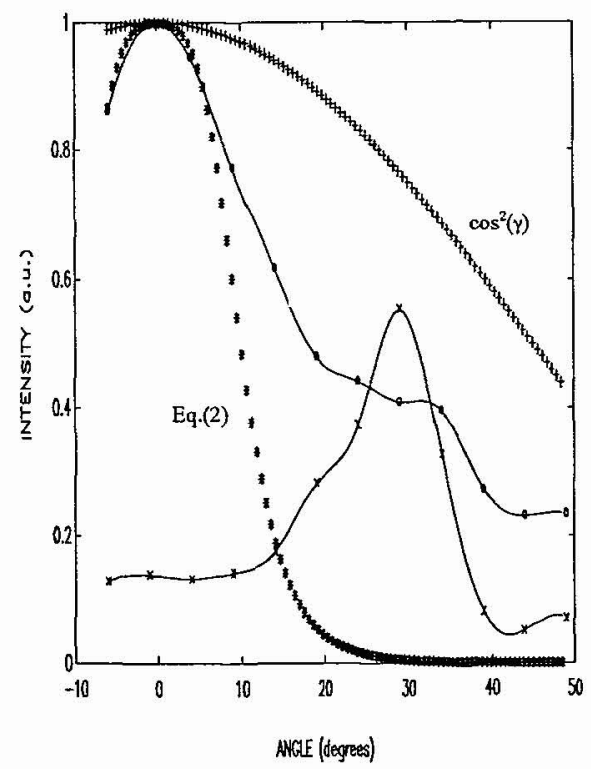

Fig.4 Photoluminescence vs $\gamma$ for two QWs: o - antinode, $x$ - node position 